\title{
Ticked off: A case of a tick involving the lower eyelid
}

\author{
Kah Wai Lai*; Dawn K. A. Lim, MBBS, MRCP (UK), MMed (Int Med), MMed (Ophth) ${ }^{*}$; \\ Blanche X. H. Lim, MBBS, MMed (Ophth) ${ }^{*}$; Yew Sen Yuen, MBBS, MMED (Ophth) ${ }^{*}$;

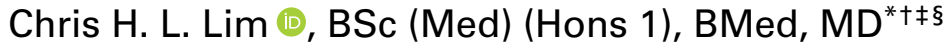

Keywords: Emergency, ophthalmology, tick

\section{INTRODUCTION}

Tick bites are not uncommon presentations to the emergency department. However, to this day, they alarm both the physician and patient alike. In addition to requests for assistance in removing these arachnids, patients may also seek medical assistance for a host of associated complications. In patients presenting with tick infestations of the eye and its associated adnexa, certain ophthalmic considerations are crucial. The lack of familiarity in the management of these patients may result in less than desirable outcomes. We therefore present a case of a young girl with a tick latched onto her eyelid and discuss salient features in the management of this affliction.

\section{CASE PRESENTATION}

A 3-year-old girl presented to the emergency department with her mother who reported concerns over a lower eyelid lesion that had been increasing in size over a 3-day duration. Her mother had noticed scattered dark perilesional circular objects that were increasing in number as well. The girl was otherwise constitutionally well with no significant perinatal or pediatric history. Apart from a recent history of outdoor activities, there was no further known history of periorbital injury or recent travel.
In the consultation room, our patient was notably fretful and apprehensive about engaging with the examining physician. However, she was able to fix and follow a visual target with no objection to occlusion. Pupillary reactions were briskly reactive with no relative afferent pupillary defect. A small, well-circumscribed greycolored lesion was identified involving the lateral aspect of her right lower eyelid, accompanied with multiple smaller dark lesions. This was identified macroscopically as a tick with its eggs and was associated with a small area of lower eyelid ulceration with bleeding proximal to the lid margin (Figures 1 and 2).

Given the challenges faced in performing a thorough examination and removal of the identified arachnid, an examination under sedation facilitated by intramuscular ketamine $(3 \mathrm{mg} / \mathrm{kg})$ was performed. No further involvement of the ocular surface was established on anterior segment examination. We proceeded with copious irrigation using topical chlorhexidine $0.05 \%$ (Medilock Medical Ltd, Oldham, UK) after instillation of topical tetracaine $0.5 \%$ (Bausch \& Lomb, Rochester, NY). Thereafter, toothed forceps were used to grasp the body of the tick and gently disengage its hypostome from the skin surface. Further irrigation with chlorhexidine was performed and the eggs removed. Her eyelids were everted, and fornices inspected and swept with cotton swabsticks to ensure the removal of residual foreign bodies. An otoscopy and inspection of the nares were also undertaken to ensure that no further ticks or their offspring were present. Following this procedure, topical tobramycin $0.3 \%$ ointment (Alcon Laboratories, Inc., Fort Worth, TX) was applied. Subsequent follow-up

From the ${ }^{*}$ Yong Loo Lin School of Medicine, National University of Singapore, Kent Ridge, Singapore; ${ }^{\dagger}$ Department of Ophthalmology, National University Health System, Singapore; ${ }^{\ddagger}$ School of Optometry and Vision Science, University of New South Wales, Sydney, NSW, Australia; and the ${ }^{\S}$ Singapore Eye Research Institute, Singapore.

Correspondence to: Dr. Chris Hong Long Lim, Department of Ophthalmology, National University Health System, 1E Kent Ridge Rd, Singapore 119228; Email: chrislimmd@gmail.com.

(c) Canadian Association of Emergency Physicians

CJEM 2020;22(2):257-260

DOI 10.1017/cem.2019.466 


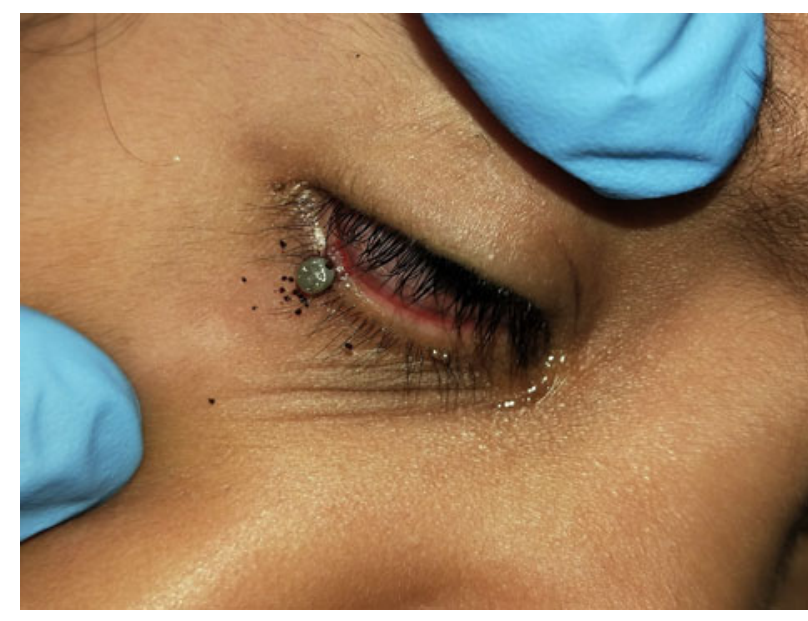

Figure 1. Clinical photograph of tick attached to the right lower eyelid with its eggs scattered on the skin surface with an associated area of ulceration.

with our patient was uneventful, and she was discharged from our service.

The tick was intact on gross examination (Figure 3) which was confirmed microscopically. This was identified to be of the Ixodidae species. In consultation with our microbiologist, no further testing or treatment was required.

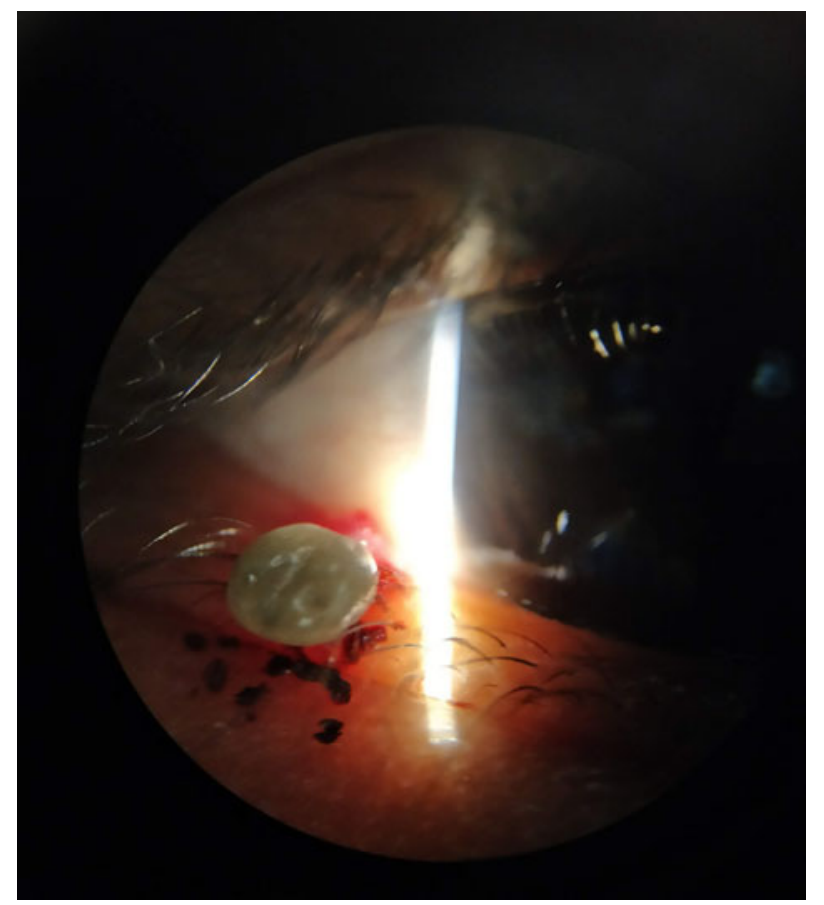

Figure 2. Slit-lamp biomicroscopy appearance of the tick and its eggs.

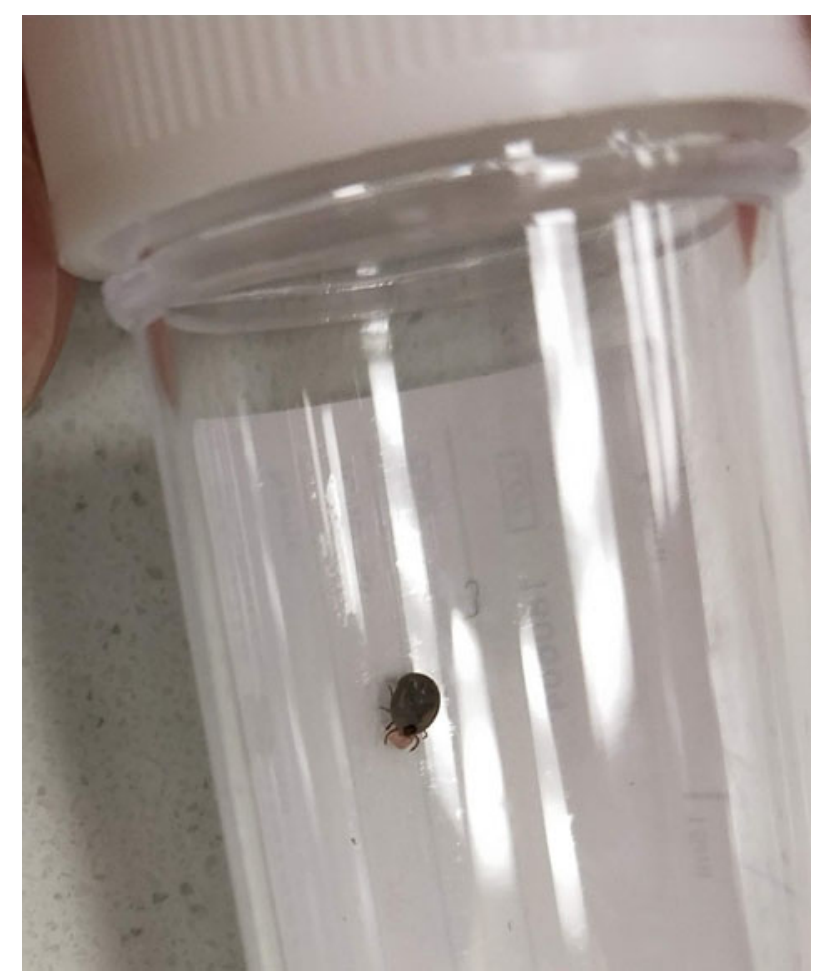

Figure 3. Clinical photograph of extracted tick.

\section{DISCUSSION}

Ticks are a ubiquitous species that have been identified across various continents, including North America, Europe, Africa, and Asia. ${ }^{1,2}$ Although local sequelae of tick bites usually resolve upon removal of the arachnid, appropriate forethought should be given to the management of these patients to manage any associated complications. A thorough history and detailed physical examination are useful to guide ongoing management.

Of the myriad of complications associated with a tick bite, the most devastating and immediate systemic complications include those of anaphylaxis, which have been reported. ${ }^{3}$ More specific concerns include envenomation with neurotoxins, which can cause ascending paralysis, respiratory failure, and death. ${ }^{4,5}$ These manifestations are life-threatening and should be promptly identified and managed in the emergency setting.

Of immediate interest in this presentation would be the exclusion of injuries to the globe and its adnexa. This may arise directly at the site of trauma and can be associated with bleeding and ulceration, as in our patient.

A breach of the skin barrier permits the introduction of pathogens, which can result in cellulitis. ${ }^{6}$ The removal of the tick should be done gently and carefully to ensure 
that no remnants are retained, as this may act as a nidus for subsequent infections in the form of preseptal or orbital cellulitis. The application of liquid butane has been reported to asphyxiate ticks and assist in the relaxation of its hypostome. ${ }^{7}$ In our patient, the application of topical chlorhexidine $0.05 \%$ may have served a similar purpose.

After removal of the tick, patients should be monitored closely for the development of secondary ocular complications, such as preseptal cellulitis and orbital cellulitis. Patients with preseptal cellulitis typically present with periorbital swelling and associated erythema, tenderness, and warmth. This may be associated with mild conjunctival injection and chemosis. Of concern for orbital cellulitis would be the presence of severe chemosis and conjunctival injection, proptosis, pain, and limitation of ocular motility. This may be accompanied by signs of optic nerve dysfunction, including a reduction in visual acuity, dyschromatopsia, and the presence of a relative afferent pupillary defect. Patients may also be constitutionally unwell and febrile. This diagnosis may be further supported by dedicated orbital imaging and represents a life and sight-threatening ophthalmic emergency. The prompt commencement of systemic broad-spectrum antibiotics should be achieved. Patients also require an admission to the hospital for further monitoring and management.

The ocular surface should also be carefully inspected for the presence of retained foreign bodies, which can inflict further trauma to delicate structures, such as the cornea and can even result in secondary infections. Although direct injuries to the skin surface typically resolve, resulting scarring may lead to eyelid malpositioning, such as ectropion. Consequently, eyelids may be unable to protect the ocular surface while malpositioned eyelashes can further damage the ocular surface.

Nonspecific local complications, including contact dermatitis, granuloma formation, allergic reactions, and secondary infections, are further associated with tick bites. ${ }^{8}$ These conditions may manifest in a delayed fashion, and patients should be further advised to return for an evaluation if signs and symptoms suggestive of these complications arise.

An important consideration in this instance would include vector-borne diseases. Because these are usually region- and species-specific, tick-borne diseases should be considered based on locality and antecedent travel history. Conditions such as Lyme disease are important considerations in temperate regions, whereas other conditions such as severe fever with thrombocytopenia syndrome may be more relevant in Asia. ${ }^{2,9}$ However, given climate change and the relative ease of travel, diseases may not be contained within regions, and careful consideration needs to be provided surrounding associated conditions. ${ }^{10}$ Patients should be warned about the development of commonly associated systemic symptoms such as a rash, fatigue, muscle aches, and fevers. Further ophthalmic manifestations of Lyme disease may include the presence of a follicular conjunctivitis, nummular keratitis, and uveitis. ${ }^{11,12}$ Cranial nerve involvement has also been reported. ${ }^{13,14}$ Discussions with the infectious diseases team regarding antimicrobial prophylaxis and further follow-up may be appropriate.

\section{CONCLUSION}

In conclusion, there are complex considerations required by physicians in the evaluation and management of patients with tick bites involving the eye and its adnexa. These considerations include possible immediate and delayed complications that may result from such a presentation. Appropriate forethought will facilitate optimal management of patients presenting with tick bites, particularly those involving the eye and its associated structures.

Competing interests: None declared.

Ethics approval and consent to participate: Consent to clinical photography for research and publication was obtained from the treated patient's guardian.

Financial support: The authors have not received any specific grants from funding agencies in the public, commercial, or not-for-profit sectors.

\section{REFERENCES}

1. Hu LT. Lyme disease. Ann Intern Med 2016;164:ITC65ITC80.

2. Kilpatrick AM, Dobson AD, Levi T, et al. Lyme disease ecology in a changing world: consensus, uncertainty and critical gaps for improving control. Philos Trans $R$ Soc B Biol Sci 2017;372:20160117.

3. McGain F, Welton R, Solley GO, Winkel KD. First fatalities from tick bite anaphylaxis. 7 Allergy Clin Immunol Pract 2016;4:769-70.

4. Hall-Mendelin S, Craig SB, Hall RA, et al. Tick paralysis in Australia caused by Ixodes holocyclus Neumann. Ann Trop Med Parasitol 2011;105:95-106. 
5. Morshed M, Li L, Lee M-K, et al. A retrospective cohort study of tick paralysis in British Columbia. Vector Borne Zoonotic Dis 2017;17:821-4.

6. Arslan F, Karagoz E, Zemheri E, Vahaboglu H, Mert A. Tick-related facial cellulitis caused by Francisella tularensis. Infez Med 2016;24:140-3.

7. Santos-Bueso E, Calvo-Gonzalez C, Diaz-Valle D, Benitez-del-Castillo J, Garcia-Sanchez J. Eyelid tick bite. Arch Soc Esp Oftalmol 2006;81:173-5.

8. Lin Y-H, Yeung L, Sun C-C, Ku W-C, Yang J-W. Contact dermatitis on the lower eyelid related to tick infestation: a case report in Taiwan. Taiwan 7 Ophthalmol 2016;6:201-3.

9. Luo L-M, Zhao L, Wen H-L, et al. Haemaphysalis longicornis ticks as reservoir and vector of severe fever with thrombocytopenia syndrome virus in China. Emerg Infect Dis 2015;21:1770.

10. Ogden N, Gachon P. Climate change and infectious diseases: what can we expect? Can Commun Dis Rep 2019;45:76-80.

11. Ehntholt MS, Zaidman GW. Unilateral diffuse nummular infiltrates and microcystic edema secondary to Lyme disease in the pediatric population. Invest Ophthalmol Vis Sci 2014;55:2828.

12. Howlett JM, Booth AP. Ocular inflammation as a manifestation of Lyme borreliosis. BM7 2012;345; epub.

13. Halperin JJ. Nervous system Lyme disease. Curr Infect Dis Rep 2015;17:445.

14. Träisk F, Lindquist L. Optic nerve involvement in Lyme disease. Curr Opin Ophthalmol 2012;23:485-90. 